\title{
Improve the students' mathematical thinking
}

\author{
Jixia Deng \\ Marine college of Shandong jiaotong university, Weihai , Shandong, China
}

Keywords: improving mathematical ability

\begin{abstract}
Mathematics learning is an intellectual activity, in the process of mathematics learning, not only to learn mathematical knowledge, but also to exercise the ability to think. Good mathematical thinking ability of a person's growth, talent, success has an immeasurable effect. So the mathematics classroom should pay attention to the training of thinking ability. This paper makes a discussion on personal experience in teaching.
\end{abstract}

\section{Introduction}

Educator Polya said: the primary responsibility of mathematics teachers is the solve mathematical problems in the book, students encounter practical problems is at a loss. Therefore, as the leading role of mathematics teachers in the classroom teaching, it is very important to actively and effectively lead students to mathematical thinking and improve the ability of students to solve problems. How to do this, the specific can be carried out from the following aspects.ability to do everything possible to solve the problem of the development of students. We often pay more attention to the past mathematics teaching to

\section{To stimulate students' interest in learning new knowledge}

Education Psychology: the study interest is the most practical internal force to promote students' learning, students' learning is the most active component of positive psychology. So to be able to focus on the students' thought and stimulate students' curiosity, so as to promote their active thinking. For example: in the explanation of the infinite recursion shrinkage geometric series all items and the formula , I first present such a problem, infinite geometric series 50, 25,12.5,6.25.. This sum of all items and if is a fixed number. Students immediately replied, "no, reason is that items in the sequence is infinite, and each is positive, numerous positive additive, and also a certain infinite, so don't can is a number。Then, I put forward second questions: A, B between the two distance is 100 meters, a from A to B, a trip is like this (Abstract), first seconds to go 100 meters of the half, second walk the rest half of the journey, the three seconds to go and then the remaining half of the journey, and so on, the number of the journey by each second is 50,25.12.5,6.25... Clearly, this sum of all items and will not be greater than 100 (m), The actual examples , students is easy to understand, it is exceeding the students' expectations, also denied the answer to the first question. At this time, to seize the curiosity of the students, the derivation of summation formula, students will be active to think, in the end is what formula so magical, can the infinite and transformed into limited. The formula is how come, not only students' thinking is stimulated and their thinking subject can actively mobilize the brain, the positive thinking, understanding and memory. This proactive exploration to find the answer, it is better than the passive acceptance of the effect, but also a happy learning, this is my best advocate. In addition to easy to remember the formula, but he also mastered how to apply, especially the actual how to apply.

\section{Break the normal procedure to break through the limitation of thinking.}

Everyone's thinking is to a certain extent, there are limitations. The limitations of this way of thinking tends to complicate a simple question, even a dead end and can not find the answer to the question. For example, there is such a problem in the textbook: seek $\lim _{x \rightarrow \infty} \frac{x+\sin x}{x}$, See the 
problem, students first think, cannot be directly applied limit four principles, types of limit is $\frac{\infty}{\infty}$, then Robida rule to find. $\lim _{x \rightarrow \infty} \frac{x+\sin x}{x}=\lim _{x \rightarrow \infty} \frac{(x+\sin x)^{\prime}}{(x)^{\prime}}=\lim _{x \rightarrow \infty} \frac{1+\cos x}{1}=\lim _{x \rightarrow \infty}(1+\cos x)$ - So

the limit does not exist. This method is wrong, wrong Robida rule applies in $\frac{\infty}{\infty}$, but and is not the type of $\frac{\infty}{\infty}$ can Robida law solution, when apply problem-solving lead to limit does not exist, does not mean that limit definitely does not exist. The correct way to solve the problem is, $\lim _{x \rightarrow \infty} \frac{x+\sin x}{x}=\lim _{x \rightarrow \infty} \frac{\frac{x+\sin x}{x}}{\frac{x}{x}}=\lim _{x \rightarrow \infty}\left(1+\frac{\sin x}{x}\right)=1+0=1$ 。 Again example : seek derivatives $y=\frac{x^{4}+x^{2}+1}{\sqrt{x}}$,

, One to see the problem is in addition to the form, the students according to the conventional method of solving the problem, First thought is using in addition to the limit derivation rule to find, although also can find out, however, the amount of calculation is too large, it is very easy to go wrong, at this time, I will guide the students, review derivative principle, law of addition and subtraction to much simpler than it was for division of the derivation rule application, for this, students are aware of, so derivative process, can turn into and form to the formation and the form, and derivative, will simplify the calculation. The simple way is to first sum form $y=\frac{x^{4}+x^{2}+1}{\sqrt{x}}=x^{\frac{7}{2}}+x^{\frac{3}{2}}+x^{-\frac{1}{2}}$

, Then according to the derivation rule and, to find quickly and not easy to make a mistake by using the derivative formula of power function. It is because of the limitations of the thinking that the students are always confined in the ready-made formulas, rules and theorems. If we teachers can timely guidance, informal in a fixed pattern, has strong adaptability, and pay attention to the special factors, thinking, students will find the best solution to the problem., and was extended to similar problems, so as the open dimension and multi solution to one problem, a method with multiple abilities.

\section{Encourage students to boldly ask questions, to overcome the inertia of thinking}

Teachers in the teaching process, don't one-sided stressed analyze and solve the problem of program or pattern, to guide the students eager to use their brains to think, ask a few why, through specific examples to guide students to observe, to obtain the conclusion. For example, in the derivation of the logarithmic function finished, I ask such a question: $(\ln 2)^{\prime}=\frac{1}{2}$, correct or not ,many students answered right, because $(\ln x)^{\prime}=\frac{1}{x}$, at this time there are individual students answer wrong, the reason is $(\ln 2)$ is a number, is a $\log$, the derivative of a number is equal to zero, so $(\ln 2)^{\prime}=0$, When the students raised the objection, the whole class is very active, then I summed up in a timely manner in the form of what is expressed in the number, its derivative is equal to zero. Students see light suddenly.Again example , In the calculation of definite integral,

there is a computational problem, first answer is

$$
\int_{-1}^{1} \frac{1}{x^{2}} d x=\left[-\frac{1}{x}\right]_{-1}^{1}=-2
$$

, Let students observe the solution to this problem is correct or not, the class are judged correctly, then I would deny their answer, prompt them according to the conditions of use of the Leibniz Newton formula to judge and 
eventually a considerable part of the students find the wrong reasons, the reason is that, Newton Leibniz formula of the conditions of use requirements of the integrand must be in the interval integral continuously, otherwise it will lead to wrong. The integrand $\frac{1}{x^{2}}$ in the integral interval [$1,1]$ is not continuous, so can not be directly applied to the formula. How to solve it? Let everybody discuss, the classroom atmosphere is very warm, very get correct solution,

$$
\begin{aligned}
& \int_{-1}^{1} \frac{1}{x^{2}} d x=\int_{-1}^{0} \frac{1}{x^{2}} d x+\int_{0}^{1} \frac{1}{x^{2}} d x \quad \text { and } \quad \int_{-1}^{0} \frac{1}{x^{2}} d x=\lim _{a \rightarrow 0} \int_{-1}^{a} \frac{1}{x^{2}} d x=\lim _{a \rightarrow 0}\left[-\frac{1}{x}\right]_{-1}^{a}=\infty, \\
& \int_{-1}^{1} \frac{1}{x^{2}} d x=\int_{-1}^{0} \frac{1}{x^{2}} d x+\int_{0}^{1} \frac{1}{x^{2}} d x=\lim _{b \rightarrow-0} \int_{-1}^{b} \frac{1}{x^{2}} d x+\lim _{a \rightarrow+0} \int_{a}^{1} \frac{1}{x^{2}} d x \\
& =\lim _{b \rightarrow-0}\left[-\frac{1}{x}\right]_{-1}^{b}+\lim _{a \rightarrow+0}\left[-\frac{1}{x}\right]_{a}^{1}=\lim _{b \rightarrow-0}\left[-\left(\frac{1}{b}+1\right)\right]+\lim _{a \rightarrow+0}\left[-\left(\frac{1}{a}-1\right)\right]=\infty \text { so } \int_{-1}^{1} \frac{1}{x^{2}} d x \quad \text { divergence. After }
\end{aligned}
$$

this explain, students are not only clear the condition of using the formula, and find the way to solve this problem, more important is that there was such an atmosphere in class, you can make students' thinking is not an inert state, mobilize the positive thinking of brain, with the passage of time, students' thinking space will become very active.

\section{Focus on the cultivation of mathematical ideas}

For example:Put interval [0,1] into three equal parts, throw away the middle of the sub interval $\left(\frac{1}{3}, \frac{2}{3}\right)$,for the rest of the two sub interval into three equal parts again, throw away the middle of the sub interval $\left(\frac{1}{9}, \frac{2}{9}\right)$ and $\left(\frac{7}{9}, \frac{8}{9}\right)$, So repeatedly to throw away the middle part of the operation. The total length of the discarded part is calculated to be 1,its length is just equal to the length of the interval $[0,1]$. the length of the remaining point is equal to 0 . clearly the rest of the point is infinite, but the total length of the remaining points is zero. They are scattered in the interval [0,1] like dust, so called the dust collection, and the number of points of this dust set actually as many as the number of the original interval, points. this is not consistent with the traditional view., the traditional view that "the whole is greater than the part", the traditional conclusion is limited to the finite interval, does not set up in an infinite interval. "The whole is greater than the part" this kind of thinking set in the infinite interval has been broken. This is the idea of mathematics.

In short, to improve the students' ability to think mathematically, the ways are various. Teachers must first improve their own quality, not only to be familiar with the teaching materials and teaching methods, but also to combine the various parts of the content, so that students grasp the overall structure of the teaching materials. Let students study, raise the ability of multi solution to one problem and multi solution to one problem training, is to guide and inspire students from different angles and different ideas, with different methods and different operation process to analyze and solve the same math exercises. Pay more attention to knowledge transfer, broaden the thinking of students in the learning process, some of the old knowledge is the foundation of new knowledge, new knowledge is the extension and development of the old knowledge. Secondly, love the students, and students mind communication, so that the students' thinking ability under the guidance of teachers, constantly improve, free development. 


\section{References}

[1] Editor, Mathematics group, Tongji University "higher mathematics” Higher Education Press

[2] Editor, Beijing Normal University, “Psychology” ,Higher Education Press

[3] School of mathematics, Sichuan University,”tan fu shu xue” 